\title{
Coaching: liderazgo y desarrollo profesional
}

Coaching: leadership and professional development

\author{
Daniel Hernán Ortiz Luzuriaga \\ Johanna Andrea Alvarez Maldonado \\ Iván Ariolfo Coronel Perez \\ Marjorie Marcela Acosta Véliz
}

Fecha de recepción: 06 de mayo de 2020

Fecha de aceptación: 20 de junio de 2020 


\section{Coaching: liderazgo y desarrollo profesional}

\section{Coaching: leadership and professional development}

Daniel Hernán Ortiz Luzuriaga ${ }^{1}$, Johanna Andrea Alvarez Maldonado ${ }^{2}$, Iván Ariolfo Coronel Perez ${ }^{3}$ y Marjorie Marcela Acosta Véliz ${ }^{4}$

Como citar: Ortiz, D., Alvarez, J., Coronel, I., Acosta, M. (2020). Coaching: liderazgo y desarrollo profesional Revista Universidad de Guayaquil. 131(2), 27-40. DOI: https://doi.org/10.53591/rug.v131i2.1354

\section{RESUMEN}

Objetivo: analizar el coaching como herramienta para el aprendizaje y desarrollo empresarial. Materiales y método: esta investigación es un estudio cualitativo documental que describe la aplicación del coaching en el ámbito empresarial y presenta resultados de éxitos en casos prácticos. Resultados: La optimización de las cualidades y habilidades empresariales es un factor fundamental para el cumplimiento de metas y desarrollo de las organizaciones. El coaching empresarial es una herramienta que incentiva al recurso humano, fomentando cambio de acciones, involucramiento en la toma de decisiones y liderazgo que se evidencia en mejora del desempeño. Es un proceso creativo y estimulante que sirve de inspiración para maximizar el potencial personal. Conclusiones: el coaching influye en la gestión del talento humano mejorando el rendimiento y el clima laboral.

PALABRAS CLAVE: Coaching Empresarial, Motivación, Formación Empresarial, Liderazgo.

\section{ABSTRACT}

Objective: To analyze the coaching as a tool for business learning and development. Materials and methods: this research is a qualitative literature review which describes the application of coaching in business and presents results of success in practical cases. Results: Optimizing the qualities and business skills is a key factor for the achievement of targets and development of organizations. Business coaching is a tool that encourages the human resource, promoting actions change, involvement in the decision making and leadership evident in performance improvement. Conclusions: Coaching influence in the management of human talent improving performance and work climate. It is a creative and stimulating process that serves as inspiration to maximize their personal potential

KEYWORDS: Business Coaching, Motivation, Business Training, Leadership

\footnotetext{
${ }^{1}$ Licenciado en Administración de Empresas, Neumann Escuela de Postgrado, Perú. Correo electrónico: danielortiz@hotmail.com

${ }^{2}$ Ingeniera en Comercio Exterior Mención Negociador Internacional, Neumann Escuela de Postgrado, Perú. Correo electrónico: andreaalvarezm@ hotmail.com ${ }^{3}$ Ingeniero en Electricidad Especialización Electrónica y Automatización Industrial, AES Panamá, Panamá. Correo electrónico: A01687158@tec.Mx

${ }^{4}$ Magister en Administración de Empresas, Universidad Rey Juan Carlos, España. Correo electrónico: m.acosta.2018@alumnos.urjc.es
} 


\section{INTRODUCCIÓN}

El estudio y evaluación del comportamiento humano crean valor en el rendimiento y éxito de la organización. Es importante determinar opciones que fortalezcan el desarrollo de las competencias del personal y la capacidad de análisis del entorno con el fin que las acciones se orienten al logro de objetivos. El desarrollo personal se basa en la capacidad de superación de problemas, mejora del desempeño y capacidad de adaptación a cambio. Las características personales que influyen en el desarrollo profesional se representan en tres niveles (Chavez, 2012):

- Primer nivel: los conocimientos y habilidades específicas.

- Segundo nivel: los valores, creencias y vocación

- Tercer nivel: las condiciones físicas, la personalidad y la inteligencia cognitiva y emocional. Los ejecutivos optimizan sus habilidades gerenciales cuando integran de forma efectiva cuerpo, mente y contenido emocional. Cuando los Gerentes experimentan dificultades para balancear trabajo y las relaciones con su entorno se evidencian problemas personales, familiares o de pareja que ocasionan stress o circunstancias que afectan su liderazgo y cumplimiento de las metas. Muchos individuos alcanzan importantes logros financieros sin sentir que han realizado su total potencial humano e inclusive perciben que han sacrificado aspectos importantes como la familia o la salud. El coaching es un mecanismo que permite integrar la vida profesional y la vida personal. Es frecuente que los profesionales desarrollen grandes habilidades intelectuales y otras áreas personales son aplazadas, tales como: recreación, participación comunitaria, salud, etc. El proceso de reflexión y visualización de una mejor forma de balancear el trabajo y la vida personal es una técnica utilizada en la práctica del coaching con el fin de evitar derivaciones negativas de una parte sobre la otra y de obtener resultados conscientes en los aspectos integrales del individuo.

El coaching es una actividad de acompañamiento que mejora el desempeño, las competencias, las actitudes, las fortalezas, las habilidades y los comportamientos de un individuo o un equipo, que es guiado por un profesional o coach que cumple un rol de consultor. Sin embargo, para Feldman \& Lankau (2005) sin una base teórica más sólida y una investigación empírica, el coaching corre el riesgo de caer en una tendencia pasajera que no tiene defensores porque no tiene evidencia (p.2).

Whitmore (citado en Chávez, 2012) reafirmó que el coaching ayuda en el proceso de aprendizaje de los individuos mediante la concientización del potencial de la persona para lograr objetivos y maximizar su desempeño. Se centra en objetivos y no en problemas, a través de acciones diferentes se logra obtener mejores resultados de los que ha obtenido hasta el momento. Este proceso permite realizar un análisis integral del cliente o coachee que reafirma sus competenciales personales y conductuales para identificar posibilidades y habilidades, logrando niveles de consciencia y de 


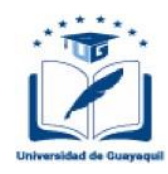

descubrimiento personal para encontrar soluciones dentro de sí, crear nuevos resultados, generar eficiencia y alcanzar objetivos deseados.

El desarrollo personal y profesional se logra a partir de la concepción mental que mejora las comunicaciones internas, lo cual se logra al elevar la conciencia, generando responsabilidad y construyendo auto confianza. El coaching favorece la optimización de las cualidades humanas para mejorar el ejercicio de las responsabilidades en la empresa, es decir, desarrollar las habilidades para lograr objetivos profesionales. El coaching ejecutivo analiza y refuerza varias competencias, como (DEMERCADEO Consulting \& Training, 2014):

- Habilidades de comunicación.

- Trabajo en equipo.

- Liderazgo.

- Aptitudes directivas.

- Capacidad de síntesis.

- Influencia.

- Motivación.

- Orientación al logro y resultados.

- Resistencia al cambio.

- Fijación y consecución de metas y objetivos.

- Orientación al cliente y vocación de servicio.

Los orígenes del coaching provienen de los años setenta, siendo aplicado inicialmente para mejorar el desarrollo profesional del tennis. Timothy Gallwey, profesor de la Universidad de Harvad, identificó que la importancia de la mente en el deporte a través del grado de conciencia que se tiene en el juego determina la diferencia entre el éxito y el fracaso; es decir, las dudas, el nerviosismo y la pérdida de concentración pueden impedir el éxito (Caby, 2012).

Las organizaciones se encuentran en constante búsqueda de mecanismos para optimizar sus recursos, desarrollar estrategias y progresar a partir de sus valores y de la misión empresarial. Las actividades empresariales demandan el desarrollo de habilidades, destrezas y competencias en el saber profesional y dominio de herramientas que se apliquen en diferentes niveles organizacionales y a diferentes necesidades u objetivos estratégicos, tales como:

- Formación de equipos de alto desempeño

- Gestión del cambio profundo

- Gestión en la crisis 
- Fortalecer habilidades gerenciales

- Mejorar la imagen directiva

- Crear habilidades de negociación

- Optimizar la planeación estratégica

- Mejorar la comunicación interpersonal.

- Resolver conflictos.

- Incrementar el nivel de compromiso con la empresa.

- Aumentar la productividad y la rentabilidad.

- Potenciar la capacidad individual en beneficio de un logro colectivo.

- Mejorar el cumplimiento y la coordinación de acciones.

- Aumentar el nivel de satisfacción laboral.

Para los empresarios es un reto constante medir acertadamente la situación de la empresa, establecer mecanismos de control y guiar el proceso de toma de decisiones para lograr el crecimiento de las organizaciones, y de ser necesario, promover el cambio estratégico. Launer (como se cita en Vidal, Cordón \& Ferrón) estableció el coaching empresarial como el proceso interactivo y directo en el cual una persona (o grupo reducido de personas) buscan el camino más eficaz para alcanzar los objetivos fijados realizando cambios profundos utilizando sus propios recursos y habilidades mediante la intervención de un agente que cumple la función de entrenador.

El coaching es una herramienta que apoya a las empresas a lograr los objetivos deseados mediante la determinación de una mayor percepción de la realidad, realizando cuestionamientos conscientes que orienten al cambio de las acciones rutinarias. Es el arte de asistir a los individuos para que logren sus objetivos, superando sus limitaciones y potenciando sus fortalezas a través de la facilitación de procesos de reflexión y toma de decisiones. El coach ayuda a las personas a identificar su situación actual y el lugar al que desea llegar para establecer objetivos y reflexión sobre el plan de acción más adecuado para lograrlos. La reflexión se logra través de preguntas para identificar y evaluar acciones, pensamientos, opiniones y resultados (Instituto de Desarrollo Empresarial, s.f.).

El coaching profesional es una técnica de entrenamiento personalizado enfocado a mejorar su actuación generando nuevas posibilidades para la acción para alcanzar resultados extraordinarios en el desempeño. Esta herramienta de gestión trabajo simultáneamente en el desarrollo personal para obtener metas tanto personales como profesionales, se orienta a cubrir el vacío existente entre donde una persona está ahora y donde se desea estar. El éxito en la evolución personal permite alcanzar objetivos profesionales y corporativos. El coach acompaña al cliente en el diseño del futuro trabajando en base de objetivos y metas (Asociación Española de Coaching, s.f.). 
La sostenibilidad de los negocios depende de las habilidades gerenciales para mantener un equipo de trabajo comprometido lo cual se logra cuando se brindan oportunidad de crecimiento profesional y estabilidad laboral. El coaching ejecutivo permite crear metas personalizadas analizando formas para cumplirlas. En este sentido, el coaching es una herramienta que facilite una transición laboral, que crea un entorno favorable para preparar a un ejecutivo en el proceso de ascenso; o fortalece nuevos comportamientos requeridos (Olvera, 2011).

El coach empresarial genera liderazgo de alto desempeño logrando equipos de alta eficiencia. Las herramientas que permiten detectar el potencial de los individuos en el lugar de trabajo crean un ambiente de innovación, liderazgo y trabajo en equipo. El proceso de coaching empresarial busca detectar las barreras internas o externas que impiden el desempeño eficiente de los individuos y formalizar el trabajo en equipo para motivar, inspirar, persuadir y potenciar las acciones que realizan en el día a día los colaboradores de una organización.

Para Muñoz \& Díaz (2014) es necesario desarrollar metodologías, como la utilización del Balanced Scored Card, para una evaluación entre los resultados generados y la inversión que se hace en un programa de coaching. El proceso de coaching transforma el entorno empresarial a través de la generación aprendizaje transformacional, ayuda a crecer a la persona en cuanto a sus relaciones interpersonales, capacidad de mejora de trabajo en equipo y un clima laboral productivo.

\section{Escuelas de Coaching}

Existen tres grandes escuelas identificadas por su origen geográfico: la Escuela Norteamericana, la Escuela Europea y la Escuela Chilena u Ontológica (Ortiz, 2010).

La Escuela Norteamericana de Coaching fundada por Thomas Leonard identifica los límites personales y diseña un entorno favorable a través del autodescubrimiento del individuo y de distinciones de principios, formas de actuar, nuevas metas o resultados. Las nuevas perspectivas o retos se fundamentan en el enfoque bajo el cual se actúa para la toma de decisiones. Por lo tanto, el rol del coach es aclarar, identificar y hacer tomar consciencia de los mecanismos bajo los cuales se han logrado los resultados actuales y se presenta desafíos de operar y tomar decisiones bajo diferentes enfoques, tales como:

- Identificación de lo urgente versus lo importante.

- Afrontar versus evitar.

- Realidad versus interpretación.

- Percepción basada en experiencias previas o paradigmas. 
- Presente o pasado.

- Dirigiéndose a versus huyendo de.

- Aceptar versus resistir

- Deseo versus necesidad

- Causa versus síntomas

- Respuesta o reacción

El objetivo del proceso de coaching bajo la Escuela Norteamérica es crear aportaciones valiosas, tales como:

- Nuevas perspectivas, opciones.

- Un mensaje, consejo.

- Solución. Un plan de acción.

- Estructura. Recurso.

- Apoyo, desafío (reto), estrategias.

- Feedback (retroalimentación).

- Aprendizaje.

La Escuela Europea de Coaching tiene sus orígenes en el sistema de aprendizaje del juego interior (The Inner Game) del norteamericano Timothy Gallwey, y se complementó con las bases de la psicología humanística. Este enfoque se basa que la diferencia entre el éxito y el fracaso no importa lo que esté sucediendo en el juego exterior, siempre hay un juego interior en la mente de las personas. La mente del jugador es quien mide la capacidad para enfrentar los obstáculos basados en los miedos o la desconfianza en uno mismo. Las experiencias previas, el nivel autoestima, las creencias religiosas y la educación general en diversos aspectos determinan las bases que el individuo utiliza para enfrentar diversas circunstancias e influyen en la toma de decisiones. En este sentido el potencial de una persona se limita por sí mismo, para incrementar al máximo su desempeño un coach debe ser capaz de identificar como el participante puede llegar a ser utilizando las siguientes acciones:

- Elevar la conciencia, mediante el autoconocimiento que lleve a la comprensión de sí mismo, aumente la seguridad e identifique el lugar y la posición al que realmente quiere llegar.

- Asumir la responsabilidad de los resultados, dejando a un lado la posición de culpabilidad. Es decir, asumir que el individuo es dueño de sus acciones y tiene la capacidad de influir en los demás sea por su forma de actuar o por las decisiones tomadas.

- Desarrollar la confianza en sí mismo para reconocerse como un ser único y valioso capaz de lograr aquello que se propone. La persona que cree en sí misma tiene confianza en lo que hace 
y en lo que es, y es capaz de rediseñarse y proponerse nuevos retos. El que cree en sí mismo tendrá una actitud más abierta al cambio y está en la búsqueda de oportunidades, para lo cual, se apoya en los demás, visualiza el futuro y reconoce su rol de importancia en la sociedad.

La Escuela Chilena de Coaching Ontológico se basa en el poder del lenguaje, observación y aprendizaje continuo en búsqueda de cambios. La observación permite identificar las cosas de una manera diferente, obtener otras percepciones para actuar diferente. Este enfoque se basa en los siguientes postulados:

- Los seres humanos como seres lingüísticos. El lenguaje establece particularidades en cada persona e influye en los fenómenos humanos.

- Poder generativo del lenguaje. La forma de hablar y sus expresiones identifica la identidad del individuo e influye en el mundo que le rodea.

- Los seres humanos se crean a sí mismos en el lenguaje y a través de él. La proyección del ser que se desea lograr se manifiesta a través del lenguaje.

El coaching ontológico se basa en el principio del desconocimiento de las cosas y la realidad individual, considerada una interpretación de cómo se observa las cosas. El cerebro interpreta lo que los sentidos perciben. No existe la certeza de que las cosas son como se dice, pues depende el grado de observación del individuo. En consecuencia, el conocimiento identifica tanto lo observado como sobre quién lo observa y se convierten en interpretaciones parciales de la realidad dando valor o limitaciones a las oportunidades, es decir se les asigna poder.

\section{MATERIALES Y MÉTODOS}

Este artículo presenta una revisión de la literatura sobre la evidencia de coaching ejecutivo. La investigación consiste en la revisión de artículos científicos en base de datos regionales Latindex, y de alto impacto como Sagepub y Emerald; además de opiniones y páginas de web de expertos nacionales e internacionales en consultoría de formación personal y profesional con el fin realizar un análisis teórico sobre el coaching y su aplicación en casos de estudios, lo cual permite identificar los beneficios y aportes para el crecimiento empresarial y el diagnóstico del sistema organizacional.

\section{RESULTADOS Y DISCUSIÓN}




\section{Entrenamiento basado en coaching}

El coach empresarial se considera a un sistema integral y continuo que desarrolla los talentos individuales de las personas, y se orienta con la medición del desempeño individual, con los resultados del equipo, compromiso por el trabajo y pasión por la excelencia. El análisis e identificación de mejores formas de ser y hacer las actividades empresariales permite determinar las acciones necesarias para generar un cambio en la organización. El coaching se instrumenta mediante la capacitación aplicando una metodología lúdica y vivencial a través de talleres y actividades de integración grupal; se estructura sistemas integrales aplicando técnicas, herramientas y tecnologías de gestión empresarial. Los objetivos que orientan estos procesos son (Conceptual Holding, s.f.):

- Desarrollar eficazmente la organización

- Generar una cultura de distinción empresarial

- Conformar las exigencias empresariales con la calidad de vida

Para Goleman (como se cita en Scott), el coaching gerencial desarrolla las fortalezas a largo plazo del recurso humano basadas en las competencias, la visión, la misión y las metas organizacionales que se reflejan en el comportamiento de los indicadores de procesos y resultados en las empresas. Esta metodología promueve la interacción estrecha entre el gerente y sus colaboradores para lo cual se puede incluir evaluaciones estructuradas, retroalimentación de 360 grados, entrevista conductual, y otras técnicas orientadas a la administración del recurso humano. Métodos complementarios de evaluación de resultados de aprendizaje y desarrollo de la organización considera la escala de autoeficacia, el compromiso organizativo, el bienestar psicológico en el lugar de trabajo y el cuestionario de liderazgo de varios factores (Lai \& Palmer, 2019).

Este proceso conduce a las organizaciones evaluar su forma de operar y se convierte en una estrategia que permite que las empresas logren resultados óptimos y mejoren su desempeño mediante el autoconocimiento de los colaboradores y directivos, la conciencia de sus prioridades y valores y el desarrollo de un mayor equilibrio personal y profesional.

El entrenamiento vivencial se basa en el desarrollo de actitudes del personal, desarrollo de habilidades, simulaciones de actividades diarias, resolución de conflictos, desarrollo de pensamiento estratégico a través de cuestionamiento y seguimiento a través de evaluación de resultados para crear hábitos de comportamiento, compromiso en crear mayor productividad y beneficios en la calidad de vida individual, de la organización y de los clientes (Salazar, 2013). Los seminarios para mandos medios están orientados para Jefes y Supervisores en un entrenamiento intensivo en liderazgo orientado a resultados; el entrenamiento al personal operativo se enfatiza el contexto de calidad y 


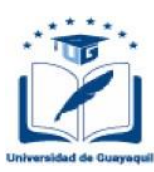

servicios proactivo con el cumplimiento de resultados; y los talleres vivenciales de team building (conformación de equipos de trabajo) se orientan a establecer consciencia de equipo y potencializar la colaboración proactiva e integración del personal (DESCUBRIR DARCON, s.f.).

El entrenamiento vivencial realizado a través de los talleres de coaching corporativo ofrece un enfoque personal orientado a resultados, que desarrolla las capacidades de influencia, relacionamiento, toma de decisiones, liderazgo y visión estratégica de sus líderes. Estos procesos incluyen el análisis y medición de la situación actual de la empresa, identificación de posibilidades funcionales para el logro de objetivos y acompañamiento en la implementación y evaluación del progreso de la visión. El diagnóstico inicial se basa en la identificación de objetivos alcanzados y objetivos deseados utilizando técnicas como evaluación de clima organizacional, análisis de competencias, entre otras técnicas de medición de gestión.

\section{Efectividad del Coaching}

El Estudio Global de Conocimiento de Consumidores 2014 del Coaching de la ICF, conducidos por PricewaterhouseCoopers, en relación con la percepción del cliente y consumidor de coaching fue realizado a 18,810 individuos de 25 países miembros y determinó los siguientes resultados:

- El 35\% describen al coaching como un proceso creativo y estimulante que sirve de inspiración para maximizar su potencial personal.

- El 33\% de las mujeres participaron en procesos de coaching para expandir oportunidades de carrera profesional

- El $42 \%$ de los hombres participaron en procesos de coaching para optimizar performance individual/equipo de trabajo

En España, en el 2009 se realizó un estudio a 40 Pymes, pertenecientes al sector servicios, sobre cómo mejorar su capacidad de gestión tecnológica, comercial y organizativa; en el cual se analizaron los efectos de la aplicación del coaching ejecutivo en los procesos de mejora dentro de las empresas. En este proyecto se realizaron diagnósticos estratégicos identificando carencias y recomendaciones para nuevos modelos de gestión de cada negocio. La metodología del estudio consistía en brindar un servicio de asesoramiento de gerencia a través de coaching en veinte empresas, mientras que en las otras empresas el proyecto finalizaba con la entrega del informe con su respectivo diagnóstico y las propuestas de mejora. Se reflejaron beneficios evidentes en las organizaciones cuyos gerentes recibieron asesoramiento tutelado frente a las que no, destacando los siguientes resultados (VidalSalazar, Cordón-Pozo, \& Ferrón-Vílchez, 2011): 
- Las actividades derivadas del coaching favorecen tanto la comprensión de las medidas de mejora propuestas como la satisfacción de los coachees. El seguimiento personalizado y la atención individualizada que los coaches prestaron a los directivos, es un factor clave que apoya al componente emocional para eliminar la resistencia hacia los cambios planteados.

- El coaching cumplió un papel de motivación y asesoramiento personalizado. El grupo que obtuvo este beneficio alcanzó un mayor nivel de comprensión de las medidas propuestas por la labor de los coaches que permite que los asesorados asimilasen los contenidos explicados.

- El grupo que sólo recibió los informes de las auditorías, tuvieron que analizar por sí mismos las conclusiones y resultados y llevar a cabo las actuaciones de mejora propuestas sin ningún tipo de ayuda en su aplicación.

- La participación en el proceso de coaching fue de mayor utilidad en la aplicación en las medidas dirigidas a mejorar las áreas de gestión (administrativa, financiera y comercial), de atención al cliente y de recursos humanos debido a la motivación y mayor comprensión de las actividades requeridas.

- La gestión del proceso de coaching no influye en cambios estructurales o a aplicación de nuevas tecnologías de la información y la comunicación, es decir, no fueron significativas entre ambos grupos. Este resultado se basa al considerable esfuerzo económico y organizativo que requieren estas medidas, lo cual se determina por la falta de recursos o el tiempo necesario para amortizar las inversiones.

\section{Casos Prácticos}

La multinacional 3M definió al coaching como una herramienta de desarrollo de negocios que se conecta directamente con su misión de ser la empresa más innovadora y el proveedor preferido en todos los mercados a los cuales sirve" (Fuenzalida, 2005). La compañía desarrolla un programa de liderazgo ejecutivo que incluye capacitación grupal y coaching individual con una orientación fuerte al cliente. El objetivo es desarrollar en los supervisores la habilidad de apoyo a los vendedores, otorgando retroalimentación y reforzando las habilidades de diagnóstico y de satisfacción al cliente.

Para la Dra. Hilda Acosta, Gerente de Áreas Esenciales de la Junta Provincial del Guayas de la Cruz Roja Ecuatoriana, el coaching empresarial le permitió un ascenso laboral, lo definió como la búsqueda de nuevos hábitos de vida e identificó que el entrenamiento del personal mejora en la producción de las empresas (Acosta, 2015). Para el Lcdo. Rubén Guzmán, Director Provincial Comunicación y RRHH, este proceso le permitió comprender la manera de pensar de otros individuos y crear empatía 


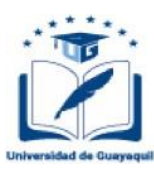

para trabajar en beneficio de la institución a través de la identificación de los mejor de cada persona y creando un clima laboral positivo (Guzmán, 2015).

El estudio de Monitor Empresarial de Reputación Corporativa- Merco 2016 determinó que el Grupo Bancolombina sea reconocido como la empresa privada de mejor Gobierno Corporativo y con mayor capacidad de retener y atraer talento en Colombia. La empresa posee la filosofía del ser para llegar al tener, para lo cual brinda a sus empleados capacitación y empoderamiento de decisiones de sus colaboradores. El coaching de Bancolombia se basa en cuatro elementos: comunicación de los líderes, entrenamiento, motivación y empoderamiento. Las actividades que se trabajan en los talleres de coaching para formación de líderes son: retroalimentación del crecimiento personal, profesional e integral; elaboración de proyecto de vida, articulación de la misión y la visión individual con la de la organización; dinámicas de habilidades, definición de roles y responsabilidades; y liderazgo (Vives, 2016).

En el año 2008 se implementó el proceso de coaching empresarial en la compañía La Fabril, empresa ecuatoriana que se dedica a la fabricación de aceite y grasa vegetales, productos de cuidado personal y aseo; y producción de insumos para pastelería, galletería, panificación, conservas entre otras El mencionado proceso se direccionó hacia la fuerza de ventas para aumentar el número de ítems por ventas y clientes por vendedor, logrando un incremento del $20 \%$ de las ventas a nivel nacional. Como resultado de esta implementación se identificó falencias con el departamento de suministros, por lo cual, se aplicó una segunda etapa de coaching empresarial enfocada a la cadena de valor que incluyó los procesos de compra, producción, distribución y calidad. Se obtuvo un incremento en el nivel del servicio del $60 \%$ al $90 \%$ (Altamirano, 2013).

Por otra parte, en el año 2010, el Banco Interamericano de Desarrollo implementó la iniciativa “Coaching for Results” en el Ministerio de Salud Pública y Asistencia Social (MSPAS) de la República de Guatemala, la cual tuvo como objetivo la optimización del proceso de adquisiciones, específicamente la modalidad de cotizaciones, y el fortalecimiento del proceso de planificación de las adquisiciones y contrataciones. Los principales cambios detectados fueron: a) evidencia inmediata de la mejora y eficiencia en los procesos; y b) una mejora en el trabajo en equipo y de la comunicación en la institución. Como principales áreas beneficiadas por el programa, surgieron las siguientes: optimización el proceso de adquisiciones al ser eliminadas tareas que no proporcionaban un valor agregado al proceso y se generaron herramientas y capacidades de gestión que permiten tener un proceso de adquisiciones más eficiente (Johnson, Morales, Pertile, \& Diego, 2011, pág. 29).

\section{CONCLUSIÓN}




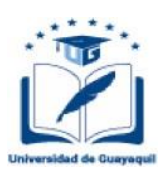

El coaching es un proceso interactivo que mejora el desempeño, las competencias, las actitudes, las fortalezas, las habilidades y los comportamientos de un individuo o un equipo. Desde el punto de vista organizacional, se desarrolla a través de actividades de acompañamiento, motivación, asesoramiento y reflexión de la cultura institucional. En consecuencia, se busca el autoconocimiento de los colaboradores y directivos, fomentar las capacidades de liderazgo, la conciencia de prioridades y valores y la orientación al cumplimiento de objetivos.

El coach empresarial desarrolla herramientas de trabajo para potenciar habilidades específicas tales como: comunicación, ventas, creatividad, visión de equipo, sensibilización al cambio, innovación, liderazgo, administración del tiempo. El coaching ofrece herramientas y estrategias que potencian las relaciones con colaboradores, colegas, jefes, clientes, proveedores, etc. El objetivo de aplicar el coaching en las empresas es incrementar la autoestima de los individuos, que den lo mejor de sí mismos, empoderarlos para que se comprometan con la empresa y desafiar a los colaboradores a tomar acción, ser fuentes que identifiquen acciones de mejoras y creen oportunidades de negocios.

El éxito del coaching en la gestión del talento humano basa en adaptabilidad y flexibilidad a las circunstancias y necesidades de la empresa, acoplándose a la realidad individual de la organización y de los colaboradores, lo cual representa un aprendizaje más dinámico. La contribución del coaching en los procesos de aprendizaje individual, de equipo y organizacional es su orientación al cambio reconociendo que las estrategias de desarrollo se deben enfocar tanto a las personas y a los procesos. Los casos analizados muestran que los mayores beneficios se obtienen en el área de gestión comercial, administración de talento humano y procesos administrativos.

Los casos expuestos evidencias que el coaching se puede aplicar para desarrollar habilidades profesionales para individuos que se desempeñan en diversas actividades administrativas tanto en empresas públicas, privadas o instituciones sin fines de lucro.

\section{REFERENCIAS}

Acosta, H. (2015, Marzo 06). Entrenamiento Organizacional Descubrir Darcon - Cruz Roja Ecuador. Guayaquil, Ecuador. Retrieved Abril 02, 2017, from https://www.youtube.com/watch?v=QnWaM2t5lgw

Altamirano, M. (2013). Coaching Empresarial enfocado en el Mejoramiento, Rendimiento, Productividad y Clima Laboral de la empresa La Fabril (Tesis de Grado). Quito: Colegio de Administración para el Desarrollo.

Asociación Española de Coaching. (n.d.). ASESCO. Retrieved Marzo 15, 2015, from http://www.asescoaching.org/el-coaching/

Caby, F. (2012). El Coaching. México: De Vecchi Ediciones. Retrieved from 
https://books.google.com.ec/books?hl=es\&lr=\&id=YG5fK73At_4C\&oi=fnd\&pg=PA1966\&dq=artic ulos+couching+empresarial\&ots=_d9tXwUnvI\&sig=d0QFIQxgTKWi_5vH8BxJx1QhmqA\#v=onep age $\& \mathrm{q} \& \mathrm{f}=$ false

Chavez, N. (2012, julio-diciembre). La gestión por competencias y ejercicio del coaching empresarial, dos estrategias internas para la organización. Pensamiento \& Gestión, 140-161. Retrieved Mayo 15, 2017, from http://www.redalyc.org/articulo.oa?id=64624867007

DEMERCADEO Consulting \& Training. (2014, Junio). Algunas Cosas Sobre Coaching Ejecutivo. Retrieved Abril 15, 2017, from www.demercadeo.com

DESCUBRIR DARCON. (n.d.). Retrieved Abril 02, 2017, from http://www.darcontrainings.com/darconconsulting/

Feldman, D. C., \& Lankau, M. J. (2005). Executive Coaching: A Review and Agenda for Future Research. Journal of Management, 31(6), 829-848. doi:https://doi.org/10.1177/0149206305279599

Fuenzalida, R. (2005). Coaching y Liderazgo. Retrieved Mayo 15, 2017, from http://www.portaldelcoaching.com/Actualidad/Columna/columna.htm

Guzmán, R. (2015, Marzo 05). Entrenamiento Organizacional Descubrir Darcon - Cruz Roja Ecuatoriana. Guayaquil, Ecuador. $\quad$ Retrieved Abril02 2017, from https://www.youtube.com/watch?v=Jjviy6D3GTw

Instituto de Desarrollo Empresarial. (n.d.). Retrieved 05 01, 2017, from http://institutocoachingempresarial.com/

Johnson, D., Morales, U., Pertile, G., \& Diego, A. (2011). Coaching for Results. Un enfoque innovador para fortalecer el desempeño institucional. El caso del Ministerio de Salud Pública y Asistencia Social de la República de Guatemala . Banco Interamericano de Desarrollo BID. Retrieved from https://publications.iadb.org/publications/spanish/document/Coaching-for-Results-Un-enfoqueinnovador-para-fortalecer-el-desempe\%C3\%B1o-institucional-El-caso-del-Ministerio-de-SaludP\%C3\%BAblica-y-Asistencia-Social-de-la-Rep\%C3\%BAblica-de-Guatemala.pdf

Lai, Y.-L., \& Palmer, S. (2019). Psychology in executive coaching: an integrated literature review. Journal of Work-Applied Management, 11(2), 143-164. doi:https://doi.org/10.1108/JWAM-06-2019-0017

Muñoz Maya, C. M., \& Díaz Villamizar, O. L. (2014, Julio). El Coaching y la transformación organizacional: una oportunidad para las Empresas y los Coaches. Suma de Negocios, 5(spe11), 62-69. doi:https://doi.org/10.1016/s2215-910x(14)70020-5

Olvera, L. (2011, Enero-Abril). Hablemos de Coaching. INCAE Business Review, 2(1). Retrieved from https://www.academia.edu/5825521/30_InCAe_BuSIneSS_revIeW_Hablemos_de_coaching

Ortiz, M. (2010, Mayo). Psicología y Coaching: marco general, las diferentes escuelas. Capital Humano(243). Retrieved from https://dialnet.unirioja.es/servlet/articulo?codigo=3206128 
Salazar, E. (2013, Septiembre 17). Pasos de un Entranamiento Darcon Consultimg. Ecuador. Retrieved Abril 15, 2017, from ttps://www.youtube.com/watch?v=zN7C5xS4YmM

Vidal-Salazar, M. D., Cordón-Pozo, E., \& Ferrón-Vílchez, V. (2011). Efectividad del coaching en los procesos de mejora de gestión de empresas. Universia Business Review, 82-101. Retrieved from http://www.redalyc.org/articulo.oa?id=43320831004

Vives, L. M. (2016). Coaching Empresarial como herramienta que potencializa el talento humano y la productivdad organizacional. Universidad Militar Nueva Granada. Retrieved from http://hdl.handle.net/10654/14884. 